\title{
Freezing Transition and Interaction Potential in Monolayers of Microparticles at Fluid Interfaces
}

\author{
L. J. Bonales, J. E. F. Rubio, H. Ritacco, ${ }^{\dagger}$ C. Vega, R. G. Rubio, and F. Ortega* \\ Departamento de Química Física I, Facultad de Química, Universidad Complutense, 28040-Madrid, Spain
}

Supporting Information

ABSTRACT: The structure and the interaction potential of monolayers of charged polystyrene microparticles at fluid interfaces have been studied by optical microscopy. Microparticles of different sizes have been studied over a broad range of surface particle densities. The structural characterization is based on the analysis of images obtained by digital optical microscopy. From the experimental images, radial distribution functions, hexagonal bond order correlation functions, and temporal orientational correlation functions have been calculated for different monolayer states at both the air/water and oil/water interfaces. The interaction potential has been calculated from the structure factor using integral equations within
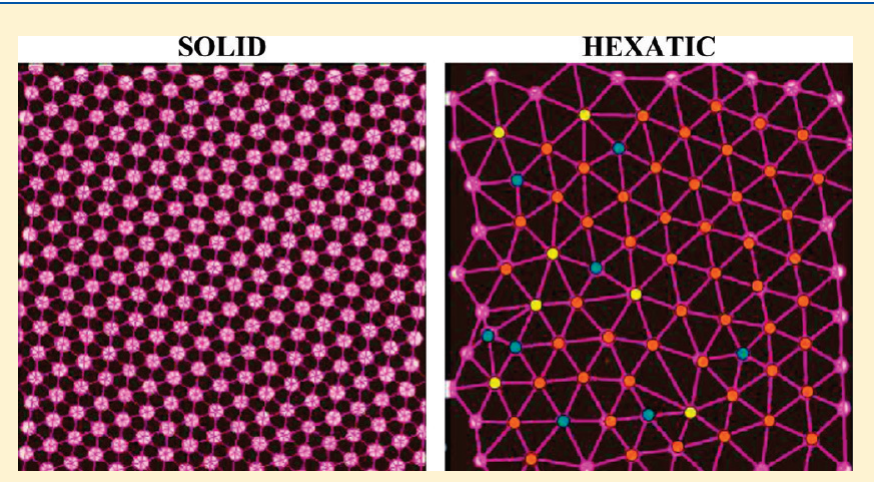
the hypernetted chain closure relationship. For particles trapped at the oil-water interface, it was found that, upon increasing the surface coverage, a freezing transition occurs, that leads to the formation of a $2 \mathrm{D}$ crystalline structure. We have studied the freezing densities of particle monolayers at the oil/water interface and compared them with Monte Carlo simulation results reported by $\mathrm{H}$. Löwen. In contrast, at the air-water interface, freezing is inhibited due to the formation of particle aggregates.

\section{INTRODUCTION}

3D colloidal systems have been recognized as model systems which can be used to understand key questions in soft matter physics. The success of this approach relies on two facts: the first one is that colloids in bulk interact through well-known potentials, ${ }^{1,2}$ and the second is that the size of the microparticles makes possible the study of these systems using relatively simple techniques.

Nowadays, the interest of colloids, more specifically, colloidal particles, is also focused on two-dimensional systems. Such systems have important practical applications like stabilization of foams and emulsions, $^{3-5}$ novel material synthesis, catalysis, wetting and dewetting, and several micro- and nanotechnological processes. ${ }^{6}$ In addition, many biological processes ${ }^{7}$ take place at interfaces, e.g., transmembrane traffic of nutrients. Wastes and metabolites are largely regulated by membrane inner and outer surfaces, which may be controlled by particles attached at the interfaces.

There are two main approaches to studying these types of systems experimentally: one is the confinement of colloidal particles between two walls, a quasi- $2 \mathrm{D}$ system, in which the particle motion is restricted to two dimensions. The other is the use of particles adsorbed at liquid interfaces. For particle-fluid contact angles close to $90^{\circ}$, the particles are trapped at the interface with a very high detachment energy $\left(\gg k_{\mathrm{B}} T\right),{ }^{8}$ which means that particles are irreversibly adsorbed onto the interface.

Almost 30 years ago, Pieranski ${ }^{8}$ reported the first $2 \mathrm{D}$ solid-like structure of polystyrene spherical particle monolayers. After evaporation of a particle dispersion, he observed with a microscope a crystalline structure with an interparticle distance much larger than the sphere diameter. This fact is evidence that the interaction between particles has a longer range than in $3 \mathrm{D}$. This was the first report highlighting the differences between the behavior of colloids in two and three dimensions.

After this discovery, several efforts have been devoted to studying the different structures that were found and the interaction potential which determines these structures. Several works report the very rich structural behavior of these $2 \mathrm{D}$ systems, such as heterogeneous structure with aggregated particles and 9 formation of homogeneous colloidal films with different degrees of order ${ }^{10,11}$ and structures with mesoscopic order ${ }^{12,13}$ (mesostructures) for which there are no equivalent structures in 3D.

One important topic in soft matter concerns the melting transition. In three-dimensional systems, this transition is wellknown. However, more than 30 years ago it was suggested that the character of the melting transition in $2 \mathrm{D}$ systems is fundamentally different from that in 3D systems. The first difference is that the fluid and solid phases have different order in two and three dimensions. Contrary to what happens in $3 \mathrm{D}$, in $2 \mathrm{D}$ the long-range positional order does not exist, and more important, in $2 \mathrm{D}$ there is another type of ordering denoted as orientational

Received: December 10, 2010

Revised: January 31, 2011

Published: March 01, 2011 
order which does not have an equivalent counterpart 3D. In summary, the structure of the particle monolayers and the interactions $^{14,15}$ between $2 \mathrm{D}$ colloids are very different from those of 3D systems.

The Kosterlitz-Thouless-Halperig-Nelson-Young ${ }^{16-19}$ theory (KTHNY) predicts that the melting transition of a $2 \mathrm{D}$ solid-like phase to a fluid phase takes place through an intermediate hexatic phase. The solid-like phase exhibiting quasi-long-range positional order and long-range bond orientational order melts to an isotropic fluid phase without orientational and positional order, through the so-called hexatic phase. The hexatic phase is predicted to have some specific characteristics: short-range positional order and long-range bond orientational order. ${ }^{20}$ The KTHNY view of the melting mechanism is that the interconversion of the $2 \mathrm{D}$ solid-like phase to the hexatic phase occurs through different defects which destroy the quasi-longrange positional order of the solid phase. The appearance of disclinations and dislocations are generated by thermal fluctuations.

Experimental studies of the melting transition in 2D are very scarce, and only validate the KTHNY theory for some systems. Zhan et al. ${ }^{20-22}$ have studied the equilibrium and dynamics of paramagnetic particles trapped at the air-water interface. By modulating the electric field (the interaction), these authors found a first-order transition from a fluid to a solid-like phase. Rice et al. ${ }^{23,24}$ have studied the melting process of poly(methyl methacrylate) and silica particles confined between two walls (quasi-2D systems), and they found that in the first system the melting process is in agreement with the KTHNY theory, while in the second it is a first-order solid-fluid transition. Lin and Chen ${ }^{25}$ found a melting transition in agreement with a KTHNY scenario for charged polystyrene particles adsorbed at the decane-water interface. All these results suggest that the freezing (or melting) mechanism seems to depend on the interaction potential between particles trapped in the $2 \mathrm{D}$ or quasi-2D environment.

The transition in 2D has been studied by Monte Carlo simulations ${ }^{26}$ for different pair potentials of the form, $u(r)=$ $\varepsilon(\sigma / r)^{n}$, where $n$ gives the range of the interaction, $r$ is the interparticle distance, $\sigma$ is the diameter of the particle, and $\varepsilon$ is the amplitude of the potential. Due to the scaling properties of this potential, it is possible to calculate structural and thermodynamic properties as a function of the rescaled density

$$
\tilde{\rho}=\left(\frac{\varepsilon}{k_{\mathrm{B}} T}\right)^{2 / n} \rho \sigma^{2}=\varepsilon^{*^{2 / n}} \rho \sigma^{2}
$$

For $n \rightarrow \infty,{ }^{27-30}$ a first-order transition with a fluid-solid coexistence, at $\tilde{\rho} \equiv \tilde{\rho}_{s}=0.887$, has been reported. For $n=12^{31}$ and $6,{ }^{32}$ a transition in agreement with a KTHNY scenario with $\tilde{\rho}_{s}=0.986$ and $\tilde{\rho}_{s}=1.513$, respectively, was found. For $n=3^{33,34}$ (corresponding to a dipolar interaction), a transition at $\tilde{\rho}_{s}=5.29$ has been found. From these results, one cannot conclude whether the solid-fluid transition in $2 \mathrm{D}$ is in agreement or not with the KTHNY theory.

In this paper, we present results on the monolayer structure of charged polystyrene microparticles, with different sizes, trapped at the air/water and oil/water interfaces, as a function of the particle surface density. The study has been carried out by digital optical microscopy, and we focused our study on the effect of the particle size on the freezing transition.
Table 1. Polystyrene Latex Particle Sizes and Three-Phase Contact Angle of These Microparticles Spread at the Air/ Water and Octane/Water Interfaces Using Isopropanol (IPA)

\begin{tabular}{cccc} 
& & \multicolumn{2}{c}{ contact angle $(\mathrm{deg})$} \\
\cline { 3 - 4 } mean diameter $(\mu \mathrm{m})$ & $\begin{array}{c}\text { standard } \\
\text { deviation }(\mu \mathrm{m})\end{array}$ & air/water & octane/water \\
1.00 & 0.04 & $121 \pm 10$ & $140 \pm 10$ \\
1.60 & 0.04 & $88 \pm 9$ & $120 \pm 12$ \\
2.90 & 0.09 & $59 \pm 2$ & $135 \pm 10$ \\
5.70 & 0.54 & $30 \pm 8$ & $120 \pm 9$ \\
\hline
\end{tabular}

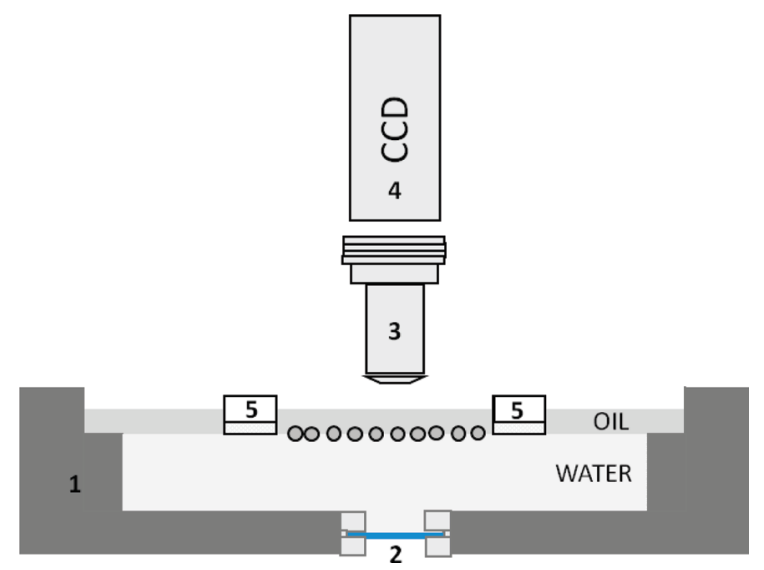

Figure 1. Setup for microscopic observation of particle monolayers at fluid-fluid interfaces. It consists of a homemade thermostatted cell (1), window for illumination from the microscope cold source (2), high AN $50 \times$ microscope objective (3), high-speed/-sensitivity CCD camera (4), and Teflon-steel barriers that control the interface area accessible to the particles (5).

\section{EXPERIMENTAL SECTION}

Materials and Chemicals. Negatively charged surfactant-free polystyrene microparticles (PS) with sulfate functional groups on the surface were provided by Interfacial Dynamics (USA); their diameters were in the $1.0-5.7 \mu \mathrm{m}$ range. Table 1 shows the characteristics of the particles. The contact angle of each particle at the interface was measured using the gel trapping technique, GTT, ${ }^{35}$ and the particle diameters were measured by dynamic light scattering (DLS). The total surface charge density of each set of particles were obtained from conductometric titration and zeta-potential, $\zeta$, measurements. As can be observed in Table 1, particles adsorbed at the air/water interface have a contact angle which decreases as the particle diameter increases; however, at the octane/water interface the contact angle remains almost constant $\left(\sim 120^{\circ}\right)$ with the particle size.

The solvents used were milli-Q water, with TOC lower than 5-2 $\mathrm{ppb}$ and resistivity higher than $18 \mathrm{~m} \Omega \mathrm{cm}^{-1}$. Octane with the highest purity available was purchased from Sigma-Aldrich and filtered through an alumina $\left(\mathrm{Al}_{2} \mathrm{O}_{3}\right)$ column before use. The spreading solvent, 2-propanol (IPA), was used as received without further purification.

Particle Tracking Microscopy. The setup (see Figure 1) is based on a Nikon Eclipse 80-I microscope with a digital head (magnification from $0.8 \times$ to $2 \times$ ) and provided with several long-distance objectives of $10 \times, 50 \times$, and $100 \times$ magnification. A CCD high-speed camera (Hamamatsu, model C8800-21C) capable of taking 30 frames per second $(\mathrm{fps})$ at full resolution $(1000 \times 1000$ pixels $)$ was used to 
record the image sequences of the particle monolayers. The monolayer was spread in a Langmuir trough (Figure 1) or in a home-built cell (see Supporting Information for the design), in both cases using a ring system to minimize the particle motions due to convection or air currents (drift). ${ }^{36,37}$ Both cells were easily housed in the microscope stage. The image sequences were stored in a computer for the subsequent analysis with homemade software.

Procedure. The particles were first redispersed as a $1-2$ wt \% suspension in water-isopropanol (IPA) $1: 1(\mathrm{v} / \mathrm{v})$ and sonicated in a cold water bath for $30 \mathrm{~min}$ to avoid any aggregates. Then, 10 to $25 \mu \mathrm{L}$ of the suspension was spread onto the interface with a microsyringe. This small quantity of IPA helps to spread the particles at the interface and then dissolves into the water phase without practically changing the interfacial tension (the spread liquid volume is less than $0.1 \%$ of the total volume of the aqueous phase). The system was kept at $25^{\circ} \mathrm{C}$ for $30 \mathrm{~min}$ before starting the experiments. Once the monolayer is prepared, the level of the monolayer is adjusted to the height of the rings set to eliminate the drift motions.

Image Analysis and Calculations. We have developed software to analyze the raw digital images and sequences and to extract the location, the time evolution of each microparticle at the interface, and several distribution and correlation functions (see details in the Supporting Information). The phase behavior and structure is usually characterized through the radial distribution function, $g(r)$. This function has been calculated from the normalized histogram, $n(\Delta r)$, of the number of particles at each radial interval, from $r$ to $r+\Delta r$, divided by the value corresponding to an ideal gas with the same particle number density, $n^{\text {id }}(\Delta r)$, for each interval

$$
g(r)=\frac{n(\Delta r)}{n^{\text {id }}(\Delta r)}
$$

The simplest approach, in which many-body interactions are completely neglected, is called the dilute gas approximation (DG). In this approach, the pair potential can be directly extracted from the radial distribution function through

$$
U(r)=-\frac{1}{\beta} \ln g(r)
$$

Unfortunately, this approach has no practical use except for very low concentrations. The radial distribution function can be related to the direct correlation function, $c(r)$, by the Ornstein-Zernike $(\mathrm{OZ})$ equation

$$
h(r)=c(r)+\rho \int h\left(\overrightarrow{\mathrm{r}}^{\prime}\right) c\left(\left|\overrightarrow{\mathrm{r}}-\overrightarrow{\mathrm{r}}^{\prime}\right|\right) \mathrm{d} \overrightarrow{\mathrm{r}}^{\prime}
$$

where $h(r)$ is the total correlation function. The inversion of $g(r)$ to extract the effective interaction potential, $U(r)$, using the $\mathrm{OZ}$ equation requires a closure relationship that in the case of Percus-Yevick (PY) and hypernetted-chain (HNC) can be expressed as

$$
c(r)=h(r)-(1-\alpha)\{g(r) \exp [\beta U(r)]-1\}-\alpha \ln \{g(r) \exp [\beta U(r)]\}
$$

where $U(r)$ is the effective pair potential and $\beta=1 / k_{\mathrm{B}} \mathrm{T}$. Setting $\alpha=0$ yields PY and $\alpha=1$ the HNC integral equations, respectively. The numerical procedure involves the Fourier transformation of eq 3

$$
\tilde{h}(k)=\frac{\tilde{c}(k)}{1+\rho \tilde{c}(k)} \mathbf{o} \tilde{c}(k)=\frac{\tilde{h}(k)}{1+\rho \tilde{h}(k)}
$$

where " $\sim$ " denotes the Fourier transform and $k$ is the wavevector. The denominator of eq 5 corresponds to the structure factor, $S(k)$. Lado has demonstrated that, in the $2 \mathrm{D}$ case, the Fourier transform $\hbar(k)$ of a function $h(r)$ with circular symmetry must be calculated by a Hankel transform ${ }^{38}$

$$
h(r)=(2 \pi)^{-1} \int_{0}^{\infty} \tilde{h}(k) J_{0}(k r) k \mathrm{~d} k \tilde{h}(k)=2 \pi \int_{0}^{\infty} h(r) J_{0}(k r) r \mathrm{~d} r
$$

where $J_{n}(x)$ is the Bessel function of the first kind of order $n$. For numerical calculations, eqs 6 are replaced by their corresponding series expansions $^{38}$

$$
\begin{aligned}
& h\left(r_{i}\right)=\frac{1}{\pi R^{2}} \sum_{j=1}^{N_{R}-1} \tilde{c}\left(k_{j}\right) \frac{J_{0}\left(k_{j} r_{i}\right)}{J_{1}^{2}\left(k_{j} R\right)} \\
& \tilde{h}\left(k_{j}\right)=\frac{4 \pi}{K^{2}} \sum_{i=1}^{N_{R}-1} c\left(r_{i}\right) \frac{J_{0}\left(k_{j} r_{i}\right)}{J_{1}^{2}\left(K r_{i}\right)} ; r_{i}=\frac{\lambda_{i}}{K} ; k_{j}=\frac{\lambda_{j}}{R}
\end{aligned}
$$

where $\lambda_{i}\left(\lambda_{1}, \lambda_{2}, \cdots, \lambda_{N R}\right)$ are the $N_{\mathrm{R}}$ positive roots of $J_{0}(x)=0$, and $R \equiv$ $r_{N R}$ and $K \equiv k_{N R}$ are the computational limits of $r$ and $k$. In contrast to the $3 \mathrm{D}$ case, the points $r_{i}$ and $k_{i}$ are not equally spaced; in fact, the spacing depends on the roots $\lambda_{i}$. The experimental $g(r)$ determines the values of $R$ and $N_{R}$, which is sufficient to obtain all the pairs $\left(k_{i}, r_{i}\right)$ since $K=\lambda_{N R} / R$. To speed up the calculations, we included within the code the first 160 roots of the Bessel function, with 15 significant digits, and the rest of the roots were used with a constant spacing of $\pi$, which introduces a maximum error of $10^{-4} \%$.

Orientational functions such as the bond orientational order parameter, $\Psi_{6}$, and the bond orientational correlation function, $g_{6}(r)$, have been calculated computing the angle between the segments connecting nearest particles using a Delaunay triangulation procedure. The bond orientational order parameter is defined by

$$
\left\langle\Psi_{6}\right\rangle \equiv \Psi_{6}=\left\langle\varphi_{6, k}\right\rangle=\frac{1}{N}\left|\sum_{k=1}^{N} \varphi_{6, k}\right|
$$

where $\varphi_{6, k}$ is calculated from

$$
\varphi_{n, k}=\frac{1}{n} \sum_{j=1}^{n} \mathrm{e}^{i n \theta_{k j}}
$$

$n$ is the number of nearest neighbors of particle $k$, and $\theta_{k j}$ is the angle between two nearest neighbor bonds in the Delaunay triangulation. The bond orientational correlation function is defined as

$$
g_{6}\left(\vec{r}-\vec{r}^{\prime}\right)=\frac{\left\langle\Psi_{6}^{*}(\vec{r}) \cdot \Psi_{6}\left(\vec{r}^{\prime}\right)\right\rangle}{\left\langle\rho(r) \rho\left(r^{\prime}\right)\right\rangle}
$$

where

$$
\begin{gathered}
\Psi_{6}(\overrightarrow{\mathrm{r}})=\sum_{k=1}^{N} \delta\left(\overrightarrow{\mathrm{r}}-\overrightarrow{r_{k}}\right) \varphi_{6, k} \\
\rho(\overrightarrow{\mathrm{r}})=\sum_{k=1}^{N} \delta\left(\overrightarrow{\mathrm{r}}-\overrightarrow{r_{k}}\right)
\end{gathered}
$$

For simplicity, we have calculated $g_{6}(r)$ as

$$
g_{6}(r)=\frac{\left\langle\varphi_{6, k}^{*}(0) \cdot \Psi_{6, k}(r)\right\rangle}{\langle\rho(r)\rangle}=\frac{1}{N}\left|\frac{\sum_{k=1}^{N} \varphi_{6, k}^{*}(0) \cdot \Psi_{6, k}(r)}{\rho_{k}(r)}\right|
$$

where $\varphi_{6, k}{ }^{*}(0)$ is the conjugate of the local bond parameter of the particle $k$.

The temporal orientational correlation function, $g_{6}(t)$, evaluates the time evolution of the orientational order, and it is calculated by

$$
\begin{aligned}
g_{6}(t) & =\left\langle\varphi_{6}^{*}(t) \cdot \varphi_{6}(0)\right\rangle \equiv\left\langle\varphi_{6}^{*}\left(t^{\prime}+t\right) \cdot \varphi_{6}\left(t^{\prime}\right)\right\rangle \\
& =\frac{1}{N n_{t}}\left|\sum_{t^{\prime}=0}^{t} \sum_{k=1}^{N}\left(\varphi_{6, k}^{*}\left(t^{\prime}+t\right) \cdot \varphi_{6, k}\left(t^{\prime}\right)\right)\right|
\end{aligned}
$$


GAS

2.1

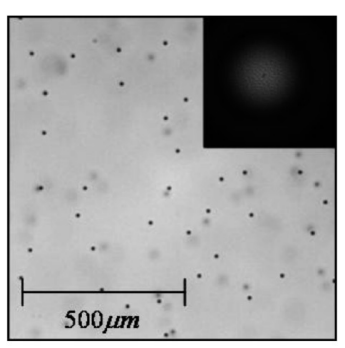

LIQUID

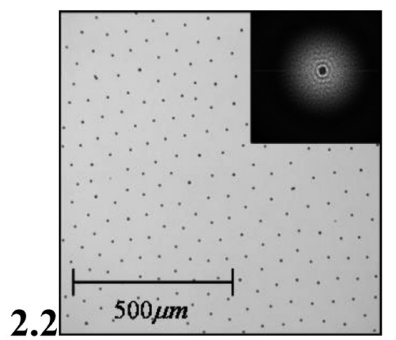

HEXATIC

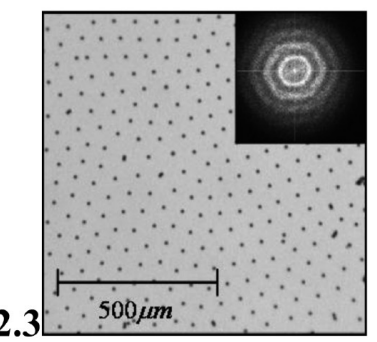

SOLID

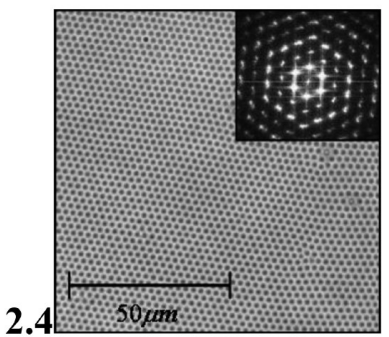

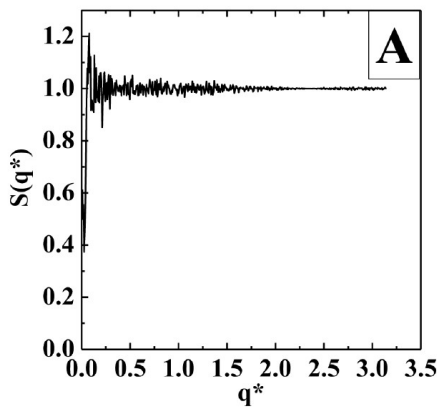
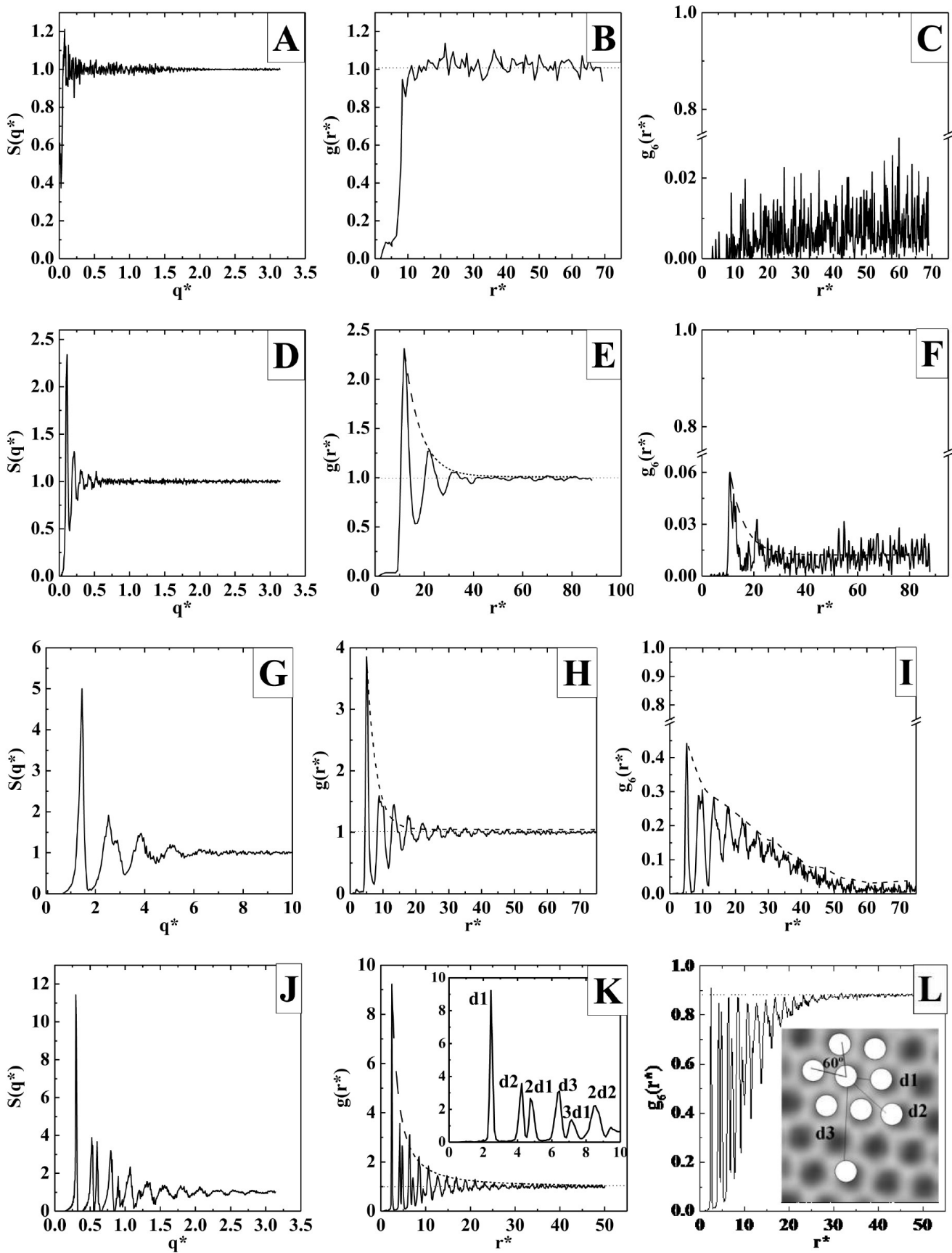

Figure 2. From left to right, examples of images, FFT images, structure factor $S\left(q^{*}\right)$, radial distribution function $g(r)$, and the bond orientational correlation function $g_{6}(r)$ corresponding to charged polystyrene particles adsorbed at the octane-water interface. The first image and its FFT image show the typical gas structure of particles with $2.9 \mu \mathrm{m}$ in diameter at a reduced surface density 0.05 ; second image corresponds to a liquid-like structure of monolayer particle of $5.7 \mu \mathrm{m}$ at 0.08 ; the third one, which shows a hexatic structure, is an image of particles with $1.6 \mu \mathrm{m}$ in diameter that has reduced surface density of $0.015 \mu \mathrm{m}$. The fourth image corresponds to a monolayer of $1.0 \mu \mathrm{m}$ particles in a perfect hexagonal array (solidlike phase).

where $\varphi_{6}(t)$ is the temporal local bond orientational function, and the star indicates the conjugate

$$
\varphi_{6, k}^{*}(t)=\frac{1}{n_{j}=1} \sum^{n} \mathrm{e}^{-i 6 \theta_{k j}(t)}
$$

As in the previous equations, $n$ is the number of nearest neighbors of particle $k$, and $\theta_{k j}$ is the angle between two nearest-neighbor bonds in the Delaunay triangulation.

\section{RESULTS}

In Figure 2, we show images of particle monolayers at the octane/water interface at different surface densities and the corresponding equilibrium functions $\left[S(q), g(r)\right.$, and $\left.g_{6}(r)\right]$. In this figure, $\rho^{*}$ is the reduced surface density $\left(\rho^{*}=\rho \sigma^{2}\right)$, where $\rho$ is the numerical density and $\sigma$ is the mean diameter), $r^{*}$ is the reduced interparticle distance $\left(r^{*}=r / \sigma\right)$, where $r$ is the interparticle distance), and $q^{*}$ is the inverse reduced distance $\left(q^{*}=2 \pi / r^{*}\right)$. 


\section{GAS}

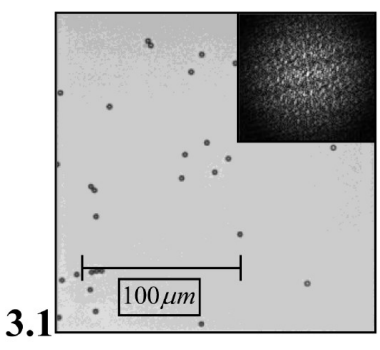

GAS

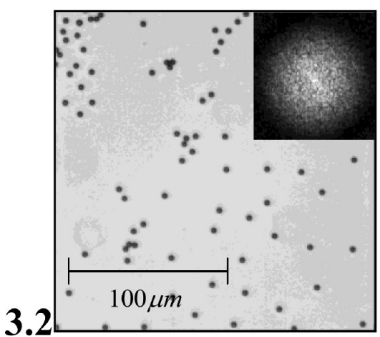

GAS

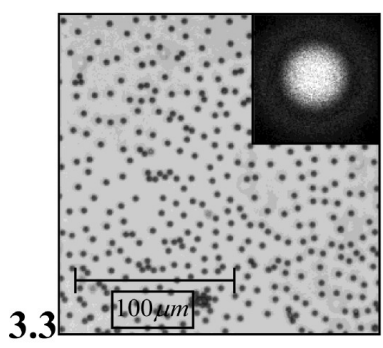

LIQUID

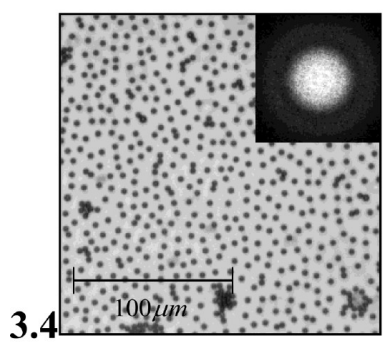

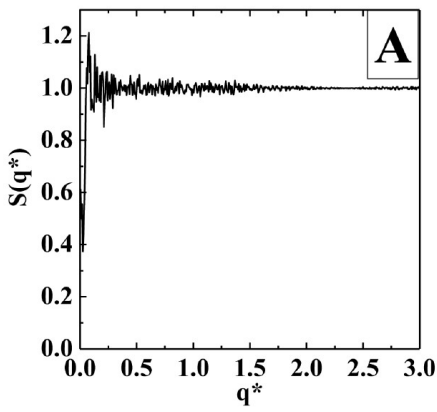
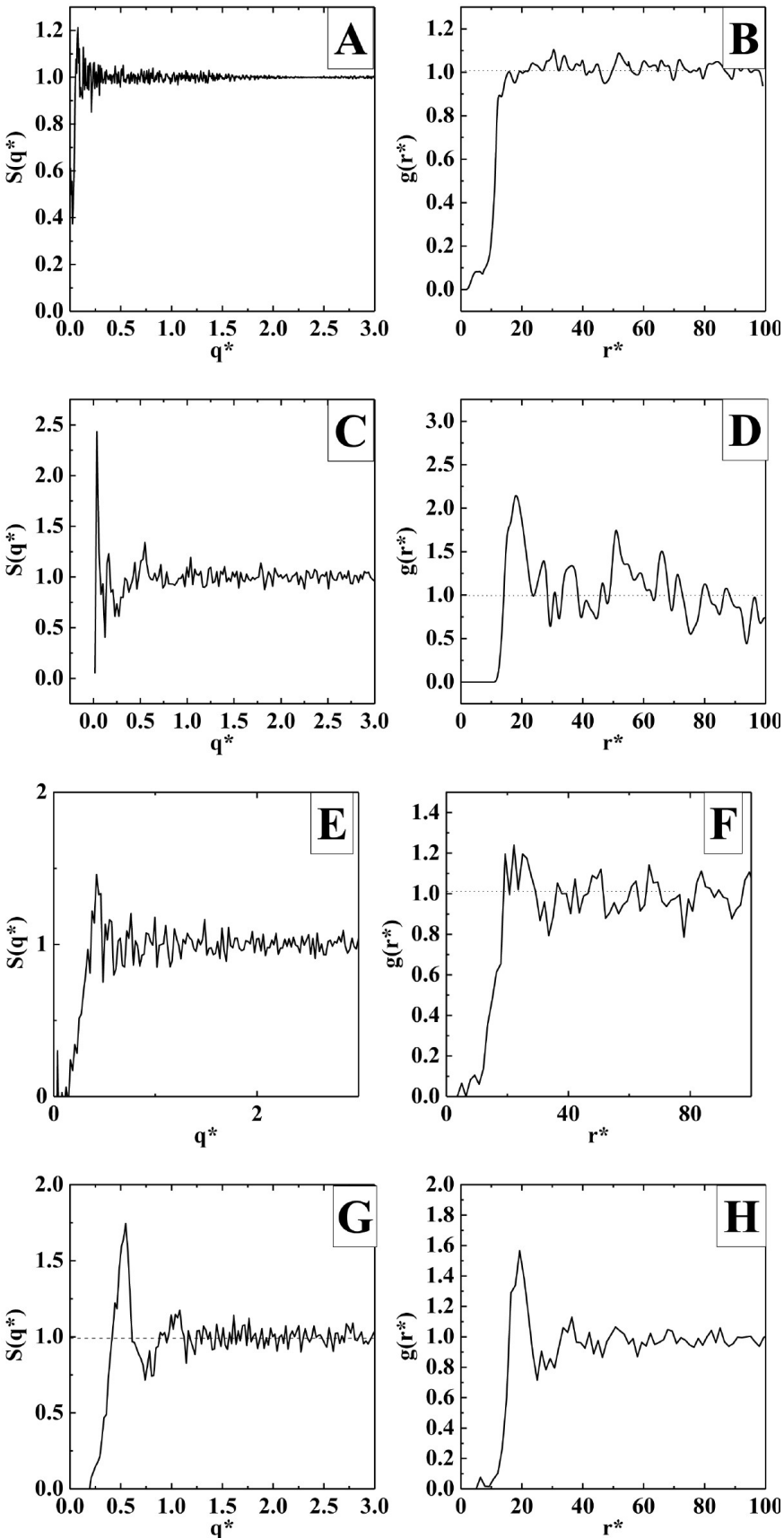

Figure 3. Examples of images, FFT images, structure factor $S\left(q^{*}\right)$, radial distribution function $g(r)$, and the bond orientational correlation function $g_{6}(r)$ (from left to right) corresponding to $2.9 \mu \mathrm{m}$ particles adsorbed at the air/water interface at four reduced surface densities: (1) 0.006, (2) 0.016, (3) 0.070, and (4) 0.110 .

Images 2.1 and 2.2 in Figure 2 correspond to fluid phases, and their structures have been characterized as gas-like and liquid-like phases, respectively. Image 2.3 corresponds to a hexatic phase structure and image 2.4 shows the typical image of a $2 \mathrm{D}$ crystalline solid with a hexagonal symmetry.

It is evident that the degree of order increases with the surface density; this is confirmed by the FFT image (inserted images in Figure 2). The FFT images corresponding to the fluid phases show a random set of points, whereas in the hexatic phase, and more clearly in the solid phase, the FFT image corresponds to a hexagonal array with a high degree of orientational order. Gaslike and liquid-like phases are characterized by the absence of orientational and positional order. The value of $g_{6}\left(r^{*}\right)$ (Figure 2c and $\mathrm{f}$ ) is zero for all interparticle distances in both phases. The $g\left(r^{*}\right)$ term on Figure 2a is a typical function for a gas, which increases from zero to unity and then remains constant; the same behavior for $S\left(q^{*}\right)$ (Figure $2 \mathrm{a}$ ) was observed for this phase. The $g\left(r^{*}\right)$ value of the liquid-like phase of Figure 2e shows a few oscillations, and its envelope decays exponentially, which indicates the fast loss of positional order when increasing the interparticle separation. $g\left(r^{*}\right)$ and $S\left(q^{*}\right)$ for a hexagonal 2D solid have typically narrow peaks with high intensity as is shown in Figures $2 \mathrm{j}, \mathrm{k}$. The positions of the maximum of each peak in $g\left(r^{*}\right)$ correspond to the characteristic distances and their multiples in a 

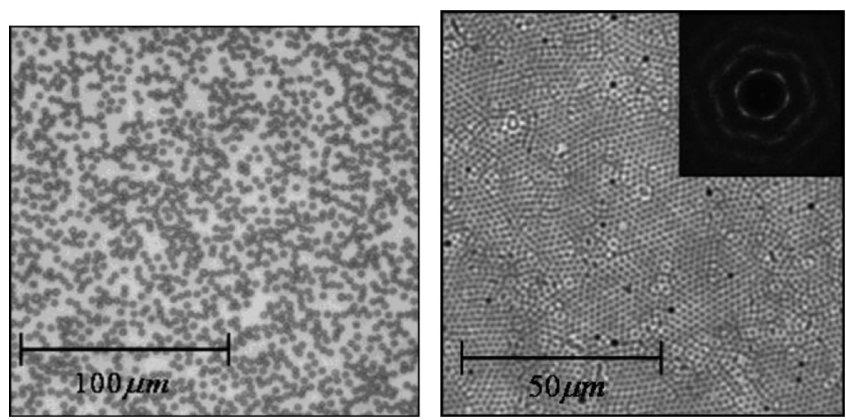

Figure 4. Examples images of aggregated particle monolayers. The left image corresponds to $2.9 \mu \mathrm{m}$ particle monolayer at the air/water interface. The right image corresponds to $1.0 \mu \mathrm{m}$ particle at the octane/water interface.

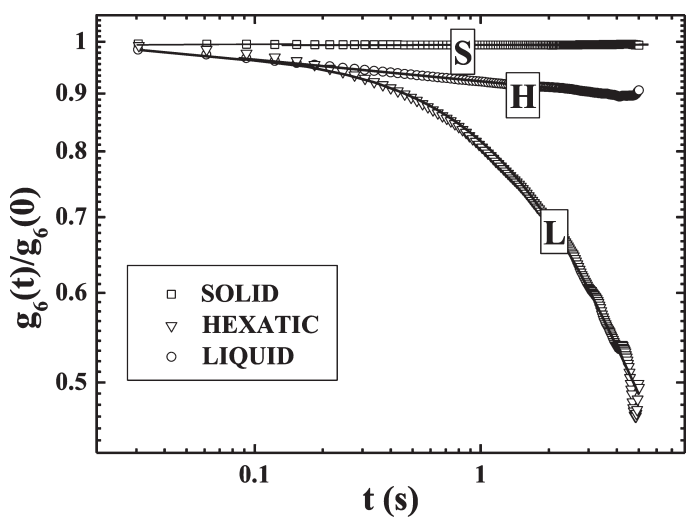

Figure 5. Bond orientational correlation function $g_{6}(t)$ normalized by the initial value of $g_{6}(t)\left(g_{6}(0)\right)$ of thee different phases corresponding to different monolayer of $5.7 \mu \mathrm{m}$ particles with structure of solid (S), hexatic $(\mathrm{H})$, and liquid $(\mathrm{L})$, and surface densities of: $2.7 \times 10^{-3}, 5.5 \times$ $10^{-4}$, and $1 \times 10^{-5}$, respectively.

perfect hexagonal array (see inset in the Figure $2 \mathrm{j}$ ). $S\left(q^{*}\right)$ shows the expected behavior in the reciprocal space. The positional order in the solid phase decays with increasing particle separation, and the $g\left(r^{*}\right)$ envelope can be fitted with an algebraically decay function. In contrast, this solid phase has a high orientational order as can be observed in the $g_{6}\left(r^{*}\right)$ function (Figure 2l) which at the maximum takes values close to one for all separation distances. This indicates that the orientational order does not decay with the interparticle distance. The hexatic phase is a phase which has orientational order but does not have positional order; this phase is characterized by oscillatory $g\left(r^{*}\right)$ and $S\left(q^{*}\right)$ functions, as in the liquid phase. The envelope of $g\left(r^{*}\right)$ (Figure $2 \mathrm{~h}$ ) decays exponentially with increasing particle separation, indicating absence of positional order. In this phase, $g_{6}\left(r^{*}\right)$ (Figure 2i) takes nonzero values at short separation distances, and its envelope decays algebraically. Note that $g_{6}\left(r^{*}\right)$ goes to zero at long separation distances, which indicates the existence of orientational order only on short ranges.

Figure 3 shows that the increase of particle density at the airwater interface induces aggregation. At low surface densities, before aggregation takes place, fluid-like phases have been observed. The FFT values for these surface densities correspond to rather disordered phases. At very low surface density, the structures correspond to a gas-like phase, where neither $g\left(r^{*}\right)$ nor

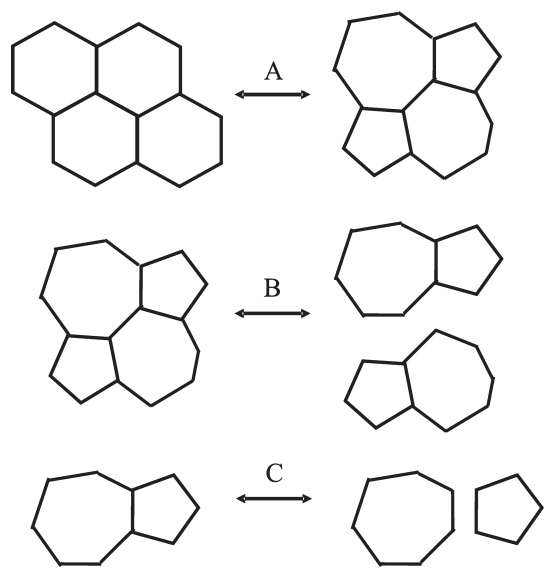

Figure 6. Sketch of the three possible equilibrium processes in the melting transition of a $2 \mathrm{D}$ solid-like phase in agreement with the KTHNY theory. The nucleation process (A) is the formation of two particles with coordination index, IC, equal to 7 , and another pair of particles with IC $=5$; the following motions induce the unbinding (B) and translation of free dislocation (C) and produce the isolated defects (16).
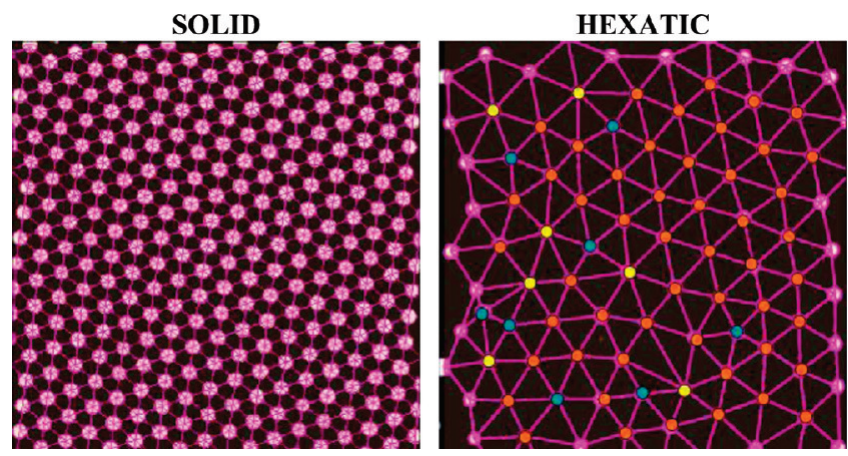

Figure 7. Examples images of solid and hexatic phases, respectively. Particles at the solid phase all have $\mathrm{CI}=6$; the different $\mathrm{CI}$ values in the hexatic phase are indicated by orange $(\mathrm{CI}=6)$, blue $(\mathrm{CI}=5)$, and yellow $(\mathrm{CI}=7)$ spheres.

$S\left(q^{*}\right)$ (Figure 3a,b,d,e,g,h) show maxima and minima and $g_{6}\left(r^{*}\right)$ (Figure $3 \mathrm{a}, \mathrm{f}, \mathrm{e}$ ) is close to zero for all interparticle distances. At higher surface densities, we have found liquid-like structures, where $g\left(r^{*}\right)$ and $S\left(q^{*}\right)$ show a few oscillations (first and second maximum) (Figure 3j,k) and $g_{6}\left(r^{*}\right)$ (Figure 3l) is close to zero for all interparticle distances. After further increase of the surface density at the air/water interface, the hexatic and the solid phases are not observed. We have found instead the formation of $2 \mathrm{D}$ aggregates (see Figure 4 left). These aggregates are also found at the octane-water interface at surface concentrations above the surface coverage corresponding to the solid phase for this interface (see Figure 4 right).

Further proof of the existence of a hexatic phase is shown in Figure 5 which illustrates the typical behavior of the $g_{6}(t)^{21}$ for particle monolayers at the octane-water interface corresponding to the different phases (solid, fluid, and hexatic). For the solid phase, $g_{6}(t)$ is constant and close to 1 , which indicates that the orientational order is maintained at long times. For fluid phases, $g_{6}(t)$ shows exponential behavior that decays to zero, which indicates the loss of the orientational order at long times. In the hexatic phase, as expected, we have found an algebraic decay in perfect agreement with the theoretical predictions. 


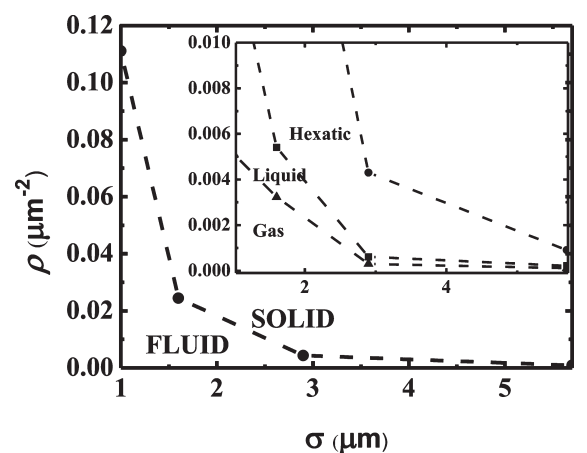

Figure 8. Phase diagram of polystyrene particles adsorbed at the octane/water interface. The different transition densities, $\rho$, are plotted against the particle diameter, $\sigma$.

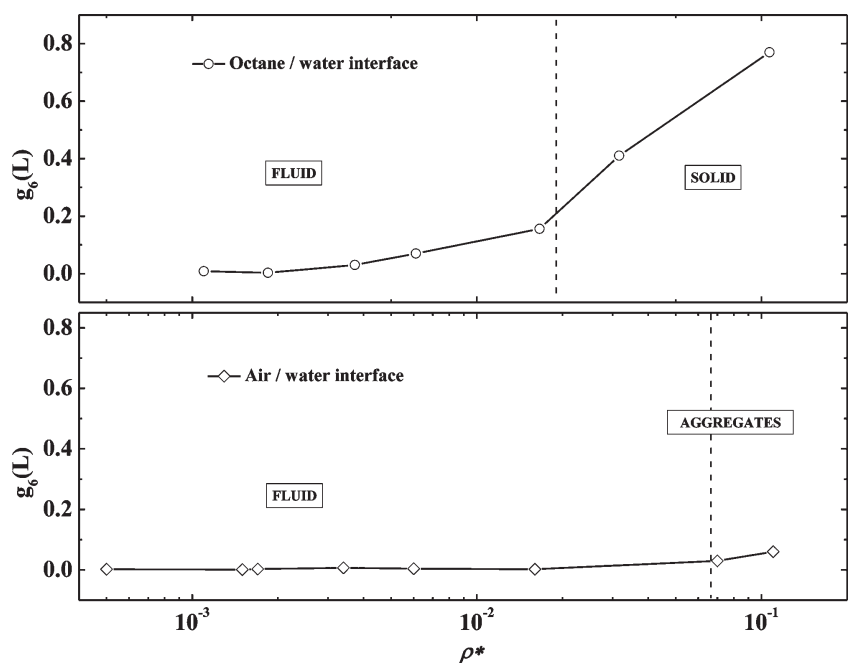

Figure 9. Phase diagrams of polystyrene particles adsorbed at the octane/water interface (upper) and air/water interface (bottom) for monolayers of particles with $2.9 \mu \mathrm{m}$ in diameter. The first maxima of the different bond orientational correlation functions, $g_{6}(L)$, are plotted against the reduced surface density, $\rho^{*}$. The vertical dotted lines indicate the fluid-solid transition densities at the octane/water interface and the beginning of the aggregation at the air-water interface.

Both the equilibrium and dynamic results of this work indicate that for the octane-water interface the melting transition follows a KTHNY mechanism. As it was mentioned in the Introduction, the KTHNY theory predicts that the melting transition consists of the interconversion of the $2 \mathrm{D}$ solid-like phase to the hexatic phase through different defects, which destroys the quasi-longrange bond positional order of the solid phase. In Figure 6, we show the details of the melting mechanism under equilibrium conditions. In each step (driven by thermal fluctuations), the motion of one or several particles induces the motion of the surrounding particles.

We have used the Delaunay triangulation to calculate the coordination index of each particle, which allows us to analyze the experimental defects found in each phase. Figure 7 shows that the defects found in all phases are in agreement with the KTHNY scenario. In the $2 \mathrm{D}$ solid-like phase, the coordination index (CI) of all particles is 6 (perfect hexagonal array). As the surface density decreases, the number of particles with $\mathrm{CI}=6$ decreases and the number of particles with $\mathrm{CI}=5$ and $\mathrm{CI}=7$ increases; i.e., in the hexatic phase showed in Figure 7, around $80 \%$ of particles have $\mathrm{CI}=6 ; 20 \%$ have $\mathrm{CI}=5$, and $20 \% \mathrm{CI}=7$. In the fluid phases, there is no preferential CI.

It is important to notice that, although we have found in our system a melting transition in agreement with the KTHNY scenario, we have also explored the possibility of fluid-solidphase coexistence. We have analyzed the particle paths of different monolayers corresponding to the different phases, gas, liquid, hexatic, and solid-like phases. For all systems, we had found homogeneous trajectories. This result means that we have pure phases. The previous statement could be wrong if the vision field of the microscope is smaller that the domain size. To disregard this possibility, we have also analyzed the local bond orientational parameter, $\Psi_{6}$, in different regions of the same monolayer, obtaining in all cases the same results within the experimental precision which confirms the absence of phase coexistence in our systems.

Phase Diagrams. Using the phase assignment described above, the phase diagrams shown in Figures 8 and 9 were constructed. In Figure 8, we show the phase diagram obtained for the octane/water interface. The fluid-solid transition density, $\rho$, is plotted against the particle diameter, $\sigma$. The transition density was calculated from the average density in 10 regions of each monolayer. Four different phase regions can be identified for all particle sizes. It can be observed that the fluid-phase region decreases when increasing the particle size. As can be seen in the inset of Figure 8, the melting transition occurs through a hexatic phase for all particles sizes. In the following, we will explain the diagram shown in Figure 8 in terms of the interaction potential.

For the sake of comparison, Figure 9 shows the values of the first maximum in the bond orientational correlation function, $g_{6}(L)$, ( $L$ being the mean distance between neighbor particles) as a function of the surface density, and for particles of $2.9 \mu \mathrm{m}$ in diameter spread onto the air/water and the octane/water interface, using IPA as spreading solvent. The vertical dot lines show the transition density calculated using the phase assignment described above. Notice that for the air/water interface at $\rho^{*}>$ 0.07 the aggregation process takes place with values for the bond orientational order parameter close to zero. However, for the same surface coverage for particles spread onto the octane/water interface the phase transition from fluid to a solid phase takes place, with values of the bond orientational order parameter close to the unity.

Freezing Density and Pair Interaction Potential, $U(r)$, at the Octane-Water Interface. In order to calculate the pair interaction potential between particles trapped at the fluid interfaces, we first restrict to low surface density monolayers, in which effective pair potentials obtained from integral equations should be closer to the real pair interaction potential. In Figure 10, as an example, we show $U(r)$ obtained for $1.0 \mu \mathrm{m}$ PS particle monolayers with low particle coverage fractions at both $\mathrm{O} / \mathrm{W}$ and $\mathrm{A} / \mathrm{W}$ interfaces. We used in this calculation the inversion scheme of the experimental $g(r)$, as was detailed in the Image Analysis and Calculations section, and the hypernetted chain (HNC) closure relationship.

The numerical values of $U(r)$ were fitted to the analytical expression:

$$
U(r)=\varepsilon(\sigma / r)^{n}
$$

As can be seen in Figure 10, the best fitting of $U(r)$ to eq 16 of all curves (represented by dashed lines in the figure) is obtained with $n=3$. This result is in perfect agreement with other 

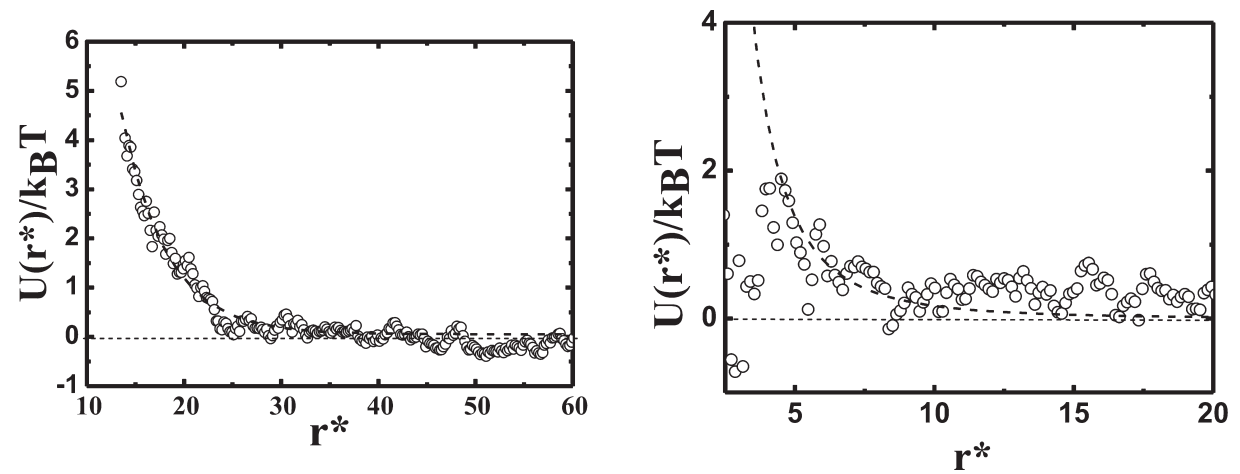

Figure 10. Reduced interaction potential $\left(U\left(r^{*}\right) / k_{\mathrm{B}} T\right)$ obtained by inversion of $g(r)$ and the HNC approximation for $1.0 \mu \mathrm{m}$ particles at the octane/ water interface (left) and $2.9 \mu \mathrm{m}$ particles at the air/water interface (right). Dashed lines correspond to a fit with a dipolar potential.
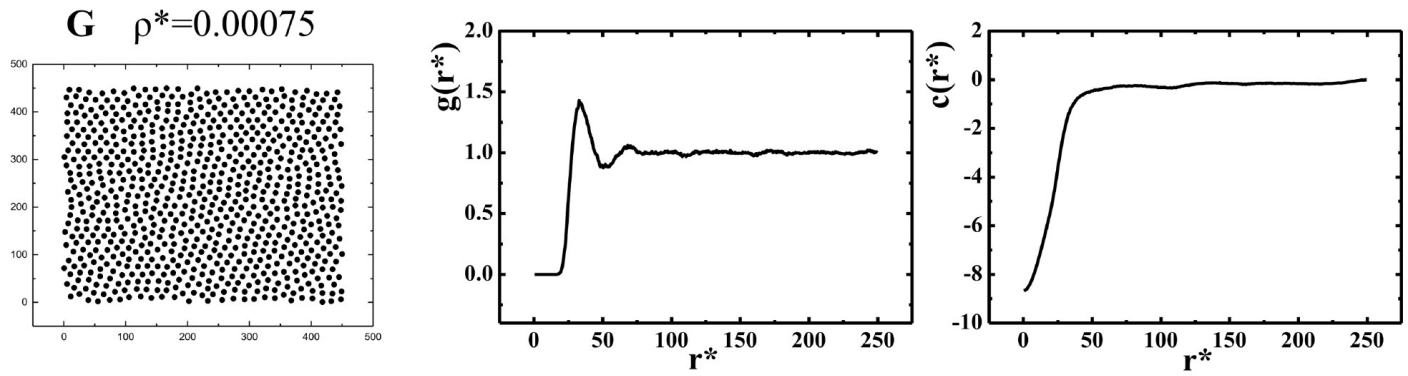

L $\rho^{*}=0.00368$

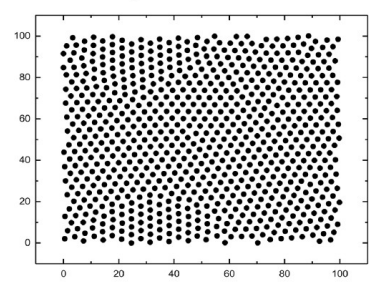

S $\quad \rho^{*}=0.1875$

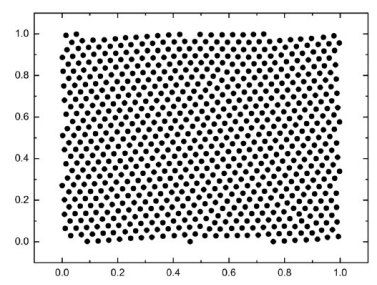

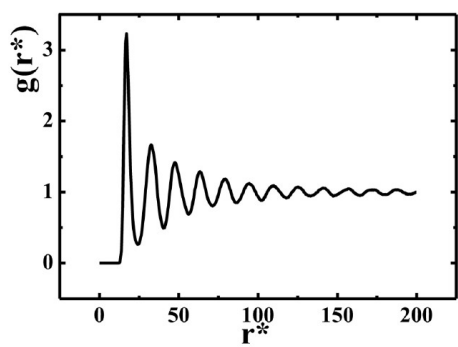
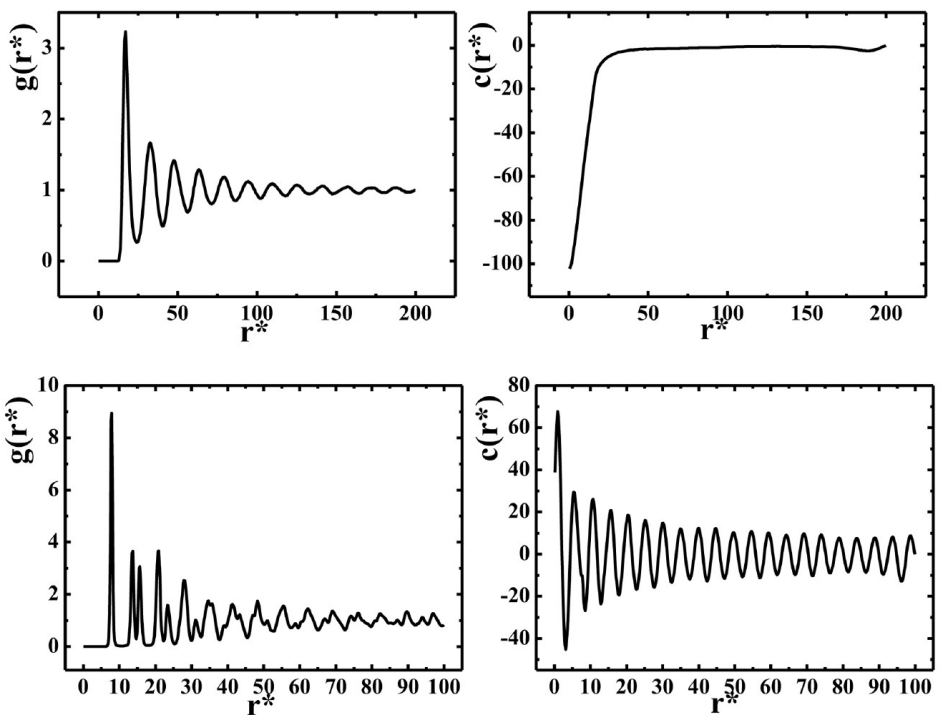

Figure 11. From left to right: configuration of a particle monolayer obtained by $\mathrm{MC}$ simulations and the equation $X$ for different phases: gas (G), liquid $(\mathrm{L})$, and solid (S). Radial distribution function $g\left(r^{*}\right)$ obtained with the PT software and the direct correlation function $c\left(r^{*}\right)$ obtained as explained in the Experimental section.

proposed models, in which the particles interact mainly through dipolar interactions $\left(U(r) \approx 1 / r^{3}\right)$. Pieranki ${ }^{8}$ proposed that the dipole-dipole repulsive interaction organizes the particles into a two-dimensional lattice. The dipole is due to the asymmetry of charge distribution on the particle adsorbed between different media; thus, electrical dipoles are associated with each interfacial particle. Averyard et al. ${ }^{11}$ proposed that Coulombic interactions could describe the behavior of particles for diluted monolayers, whereas the dipole-dipole interactions explain the behavior of particles in monolayers with high coverage fractions. The analysis of each contribution to the total interaction potential can be found in ref 39. It should be noted that, for higher densities, $\rho_{\mathrm{f}}^{*}>$ $\rho^{*} \geq 0.004$, with $\rho_{\mathrm{f}}^{*}$ being the reduced freezing particle density, we have found an effective pair potential which shows a longrange attractive component. The origin of this attractive term has been a matter of an intense debate ${ }^{40,41}$ in recent years after the work of Nikolaides et al. ${ }^{42}$ who proposed the capillary attractive force. The origin of this capillary attractive term still remains unknown, and our experiments at higher particle densities will be discussed in a forthcoming paper.

In order to check the reliability of the procedure used to calculate $U(r)$ through the inversion of $g(r)$, and for testing the different closure relationships in these systems, we have performed Monte Carlo simulations in the NAT ensemble (i.e., 


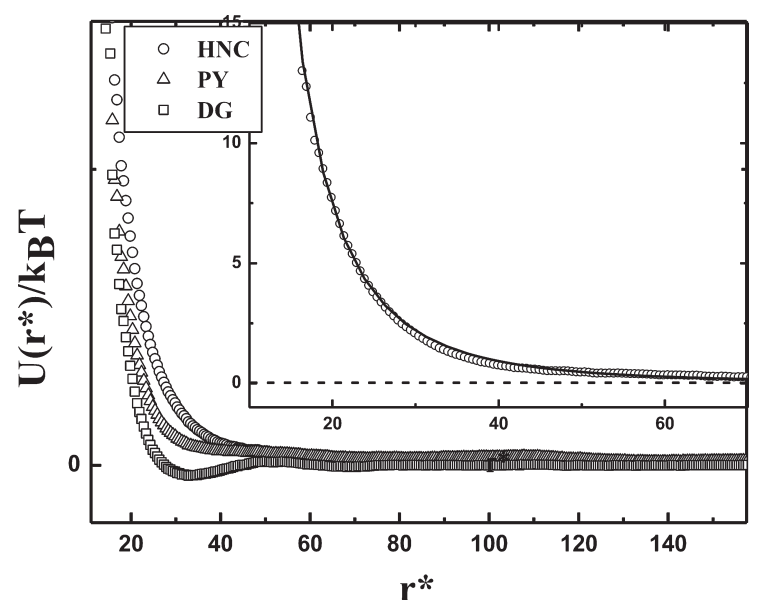

Figure 12. Inversion results of $g(r)$ of a gas phase showed in Figure 11 with DG, PY, and HNC approximations. The inset represents the best fitting (solid line) to eq 16 for a $U\left(r^{*}\right)$ obtained with the HNC approximation.

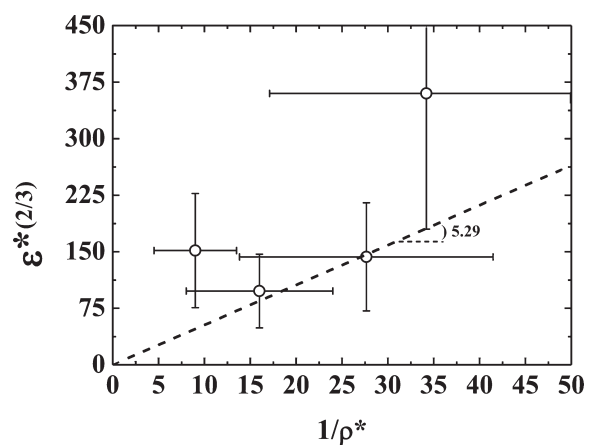

Figure 13. $\varepsilon^{* 2 / 3}$ against $1 / \rho^{*}$ (open dots) where the potential amplitude, $\varepsilon^{*}$, has been calculated as shown in Figure 9 and $\rho^{*}$ are the freezing densities obtained for particles with different sizes adsorbed at the octane-water interface. The dashed line indicates the behavior predicted by Löwen.

keeping constant the number of particles, the area, and the temperature). Different configurations corresponding to different particle densities were generated by changing the size of the bidimensional simulation box. We used $N=750$ and a repulsive dipolar potential interaction of the form $U(r)=\varepsilon^{*} / r^{3}$. Figure 11 shows the configurations generated for three different densities corresponding to gas, liquid, and solid-like phases, respectively. The radial distribution function, $g\left(r^{*}\right)$, and the direct correlation function, $c\left(r^{*}\right)$, are also shown for each phase. As can be seen in Figure 11, by using a dipolar interaction potential, it has been possible to reproduce by Monte Carlo experiments the phases found in the real systems. From $c(r)$, we have obtained the interaction potential $U(r)$ by using the other three and more simple closure relationships: diluted gas (DG), hypernetted chain (HNC), and Perkus-Yevick (PY). Figure 12 shows the interaction potential $U(r)$ obtained for the gas-like phase shown in Figure 11 for the three closures. As can be seen even for fairly low surface densities, $\rho^{*} \approx 0.00075$, which we can consider as a $2 \mathrm{D}$ gas-like system, the DG approximation fails, giving first a nonphysically attractive term at the same position of the $g(r)$ maximum, and for higher $\rho^{*}$, an oscillatory effective potential. The PY closure also fails for $\rho^{*} \geq 0.003$ leading to an oscillatory effective potential. The HNC closure leads to consistent results for all the low-density monolayers, $0<\rho^{*}<0.004$, yielding a $U(r)$ value that shows a dependence on the distance of the form $U\left(r^{*}\right)=\varepsilon^{*} / r^{* n}$ with $n=3$, i.e., a dipolar repulsive interaction potential (see inset of Figure 12). The inset in this figure shows the $U(r)$ calculated with the HNC closure relationship (open dots) and the best fits with the dipolar potential interaction (solid line), which leads to a value of the potential amplitude of $\varepsilon^{*}=$ $59000 \pm 300$, which is in perfect agreement with the potential used in the Monte Carlo simulation to generate the different configurations.

As was mentioned in the Introduction section, the transition in 2D has been studied by simulation for several interaction potentials. In the case of the dipolar interaction, $U(r)=\varepsilon(\sigma /$ $r)^{3},{ }^{33,34}$ Lowen $^{26}$ has predicted the appearance of the freezing density when $5.29=\varepsilon^{* 2 / 3} \rho^{*}$, where $\rho^{*}$ is the reduced surface density and $\varepsilon^{*}$ the potential amplitude (see eq 1 ). We have used the experimental data of the potential amplitude, $\varepsilon^{*}$ (Figure 10), and the freezing density (Figure 8 ), obtained for particles with different sizes adsorbed at octane/water interface, to check the Löwen predictions. In Figure 13, we have plotted $\varepsilon^{*^{2 / 3}}$ against $\rho^{*}$ (open dots). The straight line represents the behavior predicted for Löwen, which is in reasonable agreement with our experimental results.

\section{- CONCLUSIONS}

We have studied the freezing transition of particle monolayers at the oil-water interface as a function of the surface coverage fraction and for four particle diameters ranging from 1.0 to $5.7 \mu \mathrm{m}$. For all particles, the $2 \mathrm{D}$ phase transition from the liquidlike to solid-like monolayers occurs through a hexatic phase and is in agreement with the KTHNY theory. ${ }^{35-38}$ For each particle diameter, we have calculated the freezing density, which is different for each particle size, as found in the results obtained by using Monte Carlo simulations. We have worked out a typical dipolar potential interaction obtained by inversion of the experimental $g(r)$. This potential interaction allowed us to obtain the potential amplitude. We have also studied the behavior of the freezing surface density as a function of the particle size and found that it was in agreement with the Monte Carlo results given by Löwen. In contrast, at the air-water interface, the freezing is inhibited due to the presence of particle aggregations.

\section{ASSOCIATED CONTENT}

S Supporting Information. Cell and ring system designs and details of particle tracking software and analysis routine. This material is available free of charge via the Internet at http://pubs. acs.org.

\section{AUTHOR INFORMATION}

\section{Corresponding Author}

*Author e-mail address: fortega@quim.ucm.es.

\section{Present Addresses}

${ }^{\dagger}$ Departament of Physics, Universidad Nacional del Sur - CONICET, Av. Alem 1253 (8000) - Bahía Blanca, Argentina.

\section{ACKNOWLEDGMENT}

This work was supported in part by MICINN under grant FIS2009-14008-C02-01, by ESA under grants MAP AO-00-052 
(FASES) and PASTA, and by EU under grant Marie Curie ITNMULTIFLOW. L.J.B. was supported by a FPI fellowship and H. $\mathrm{R}$. by a Ramón y Cajal contract from MICINN. The authors are grateful to the UIRC of the CAI of Spectroscopy (Complutense University).

\section{REFERENCES}

(1) Derjaguin, B. D.; Landau, L. Acta Physicochim. URSS 1941, 14, 633 .

(2) Israelachivili, J. N. Intermolecular and Surface Forces, Academic Press: San Diego, 1991.

(3) Stocco, A. Soft Matter 2009, 5 (11), 2215.

(4) Ramsted, W. Proc. R. Soc. 1903, 72, 156.

(5) Pickering, S. U. J. Chem. Soc. 1907, 91, 2001.

(6) Dinsmore, A. D.; Hsu, M. F.; Nikolaides, M. G.; Marquez, M.; Bausch, A. R.; Weitz, D. A. Science 2002, 298, 1006.

(7) Gorter, M. D.; Grendel, F. J. Exp. Med. 1925, 41, 439.

(8) Pieranski, P. Phys. Rev. Lett. 1980, 45, 569.

(9) Onoda, G. Y. Phys. Rev. Lett. 1985, 55, 226.

(10) Horozov, T. S.; Averyard, R.; Clint, J. H. Langmuir 2004, 21, 2330.

(11) Averyard, R.; Clint, J.-H.; Paunov, V. N. Langmuir 2000, 16, 1996.

(12) Ghezzi, F.; Earnshaw, J. C.; Finnis, M.; McCluney, M. J. Colloid Interface Sci. 2001, 238, 433.

(13) Ghezzi, F.; Earnshaw, J. C. J. Phys.: Condens. Matter 1997, 9, L517.

(14) Binks, B. P., Horozov, T. S., Eds. Colloidal Particles at Liquid interfaces; Cambridge University Press: Cambridge, 2006.

(15) Marcus, A. H.; Rice, S. A. Phys. Rev. E 1997, 55, 637.

(16) Kostelitz, J. M.; Thouless, D. J. J. Phys. (Paris) 1972, 5, L124.

(17) Kostelitz, J. M.; Thouless, D. J. J. Phys. (Paris) 1973, 6, 1181.

(18) Young, A. P. Phys. Rev. B 1979, 19, 1855.

(19) Halperin, B. I.; Nelson, D. R. Phys. Rev. Lett. 1978, 41, 121.

(20) Zahn, K.; Lanke, R.; Maret, G. Phys. Rev. Lett. 1999, 82, 2721.

(21) Zahn, K.; Maret, G. Phys. Rev. Lett. 2000, 85, 3656.

(22) Zahn, K.; Méndez-Alcaraz, J. M.; Maret, G. Phys. Rev. Lett. 1997, 79, 175.

(23) Zangi, R.; Rice, S. A. Phys. Rev. E 1998, 58, 7529.

(24) Zangi, R.; Rice, S. A. Phys. Rev. E 2000, 61 (1), 660.

(25) Lin, B. J.; Chen, L. J. Colloids Surf., A 2006, 284, 239.

(26) Löwen, H. Phys. Rev. E 1996, 53, 1.

(27) Lee, J.; Strandburg, K. J. Phys. Rev. E 1992, 46 (11), 192.

(28) Zollweg, J. A.; Chester, G. V. Phys. Rev. E 1992, 46 (11), 186.

(29) Weber, H.; Marx, D. Europhys. Lett. 1994, 27, 593.

(30) Weber, H.; Marx, D.; Binder, K. Phys. Rev. E 1995, 61 (14), 636.

(31) Broughton, J. Q.; Gilmer, G. H.; Weeks, J. D. Phys. Rev. E 1982, $25,4651$.

(32) Allen, M. P.; Tildesley, D. J. Computer Simulation of Liquids; Oxford Scientific Publishers: Oxford, 1989.

(33) Amstrong, A. J.; Mockler, R. C.; O’Sullivan, W. J. J. Phys.: Condens. Matter 1989, 1, 1707.

(34) Kalia, R. K.; Vashishta, P. J. Phys. C 1981, 14, L643.

(35) Paunov, V. N. Langmuir 2003, 19, 7970-7976.

(36) Klingler, J. F.; McConnell, H. M. J. Phys. Chem. 1992, 97, 2962.

(37) Klinger, J. F.; McConnell, H. M. J. Chem. Phys. 1993, 97, 6096.

(38) Lado, F. J. Comput. Phys. 1971, 8, 417.

(39) Martínez-López, F.; Cabrerizo-Vílchez, M. A.; Hidalgo-Alvarez, R. J. Colloid Interface Sci. 2000, 232, 303.

(40) Mengens, M.; Aizenberg, J. Nature 2003, 424, 1014.

(41) Oettel, M.; Domínguez, A.; Dietrich, S. Phys. Rev. E 2005, $71,051401$.

(42) Nikolaides, M. G.; Bausch, A. R.; Hsu, M. F.; Dinsmore, A. D.; Brenner, M. P.; Weitz, D. A. Nature 2002, 420, 299. 